
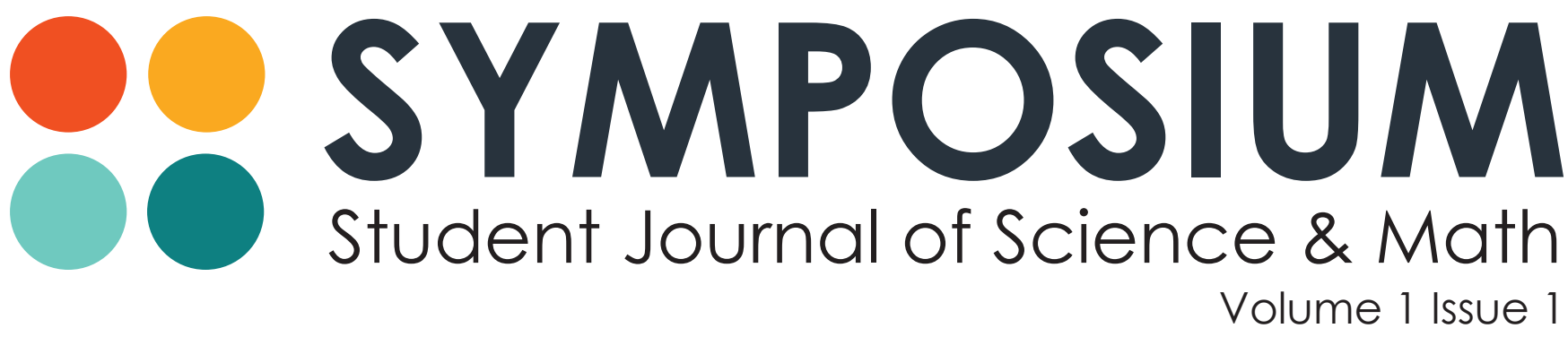


\section{CHARACTERIZING THE RESPONSE OF MULTIDRUG-RESISTANT KLEBSIELLA PNEUMONIAE SPECIES TO THE APPLICATION OF A PHAGE COCKTAIL}

A RESEARCH PROPOSAL

by Steven Liu

\section{Project Summary}

The application of bacteriophages to treat bacterial infections is known as phage therapy, which takes advantage of bacteriophage's natural ability to infect and lyse bacterial hosts. Phages have been shaped by billions of years of evolution to be highly specialized deliverers of bactericidal agents to the cytoplasm of their target bacteria. Ever since discovery of bacteriophages in 1915, phage therapy was recognized as a potentially powerful tool for eliminating bacterial infections. The effectiveness of phage therapy can be increased by creating a mixture of multiple phages to target a wider variety of bacterial strains. Furthermore, phage therapy has many advantages over antibiotics. For example, phages replicate in vivo, so a smaller effective dose can be administered. Phages will also stop reproducing once the target bacteria is destroyed. Much of the evidence strongly shows that, appropriately administered, phage therapy is effective for treatment of bacterial infectious diseases, especially those caused by multidrug-resistant bacteria.

The worldwide spread of Klebsiella pneumoniae spp., which is resistance to a variety of antibiotics, threatens to revert modern medicine to a pre-antibiotic era. Infections caused by drug-resistant $K$. pneumoniae spp. are life threatening because of our limited ability to treat the associated disease symptoms. As a result, infections due to multidrug resistant $K$. pneumoniae spp. have an extremely high mortality rate. The design of a phage cocktail with potential therapeutic application against multidrug-resistant $K$.pneumoniae spp. may enable us to respond to a rapidly emerging generation of multidrug-resistant bacterial species. 


\section{Introduction}

\section{Basics of phage biology}

Phages are naturally occurring viruses that specifically infect and lyse bacteria. The majority of reported phages are tailed phages, composed of an icosahedral head and tail, and all of them have doublestranded DNA as the genome (Matsuzaki, 2005). Phages can be further classified into two groups according to their life cycle. The lytic phage self-proliferates by intracellular development, maturation, and finally release of virions from the host cell resulting in the death of the bacteria. But the lysogenic phage integrates its phage genome into the bacterial genome, thereby allowing the phage genome to multiply synchronously with the host bacteria without destroying it. The generic life cycle of a phage begins with phage adsorption to a receptor on the bacterial surface, and then inserts phage DNA into the bacterial cytoplasm. The DNA is replicated, synthesized, assembled, and released from the bacteria (due to the lytic action of the proteins holin and endolysin), encoded by the phage genome, as shown in Figure I. (Young, 1992). Distinct phage isolates achieve antibacterial activity by differing in their mechanism of entry or strategy of replication.

\section{Phage therapy \& its advantages}

Phage therapy can be used for its lytic ability to control bacterial infection. There are multiple advantages of phage therapy over conventional chemical antibiotics. For example, there is no need to establish a dosage for phages because they are capable of replicating specifically where their hosts are located (Carlton, 1999). This eliminates the need to determine an effective dose of the drug which may be potentially dangerous if an excessive dose is administered. Only a single dose is required to achieve active therapy, resulting in greater bacterial clearance (Abedon \& Abedon, 20I0). In addition, phage therapy is minimally disruptive towards resident gut flora, unlike many chemical antibiotics, due to their host specificity. Phage host specificity also reduces the potential for inducing resistance by limiting the number of bacterial types, with which selection for specific phage-resistance mechanisms can occur (Hyman \& Abedon, 20IO; Skurnik et al., 2007; Gupta \& Yogendra, 20II). From an economic perspective, the cost of phage production is relatively low compared with chemical antibiotics. Therapeutic phage products would therefore be more readily available to the general public, in contrast to several of the more expensive antibiotics available today (Skurnik et al., 2007; Kutter et al., 20I0; Kramberger et al., 2010). The effectiveness of phage therapy can be increased by expanding the host-range of phages, because success depends largely on whether the phage can attach to the bacterial surface receptor. The host range may be limited to just a subset of strains within a single species, or it may include a broad range, spanning multiple bacterial genera (Hyman \& Abedon, 20IO).

\section{Expanding host-range with phage cocktails}

Phage infection can only occur after phage attachment to a bacterial cell receptor. Bacterial cell receptors may include components of the $\mathrm{O}$-antigen, outer core oligosaccharide of the lipopolysaccharide, and a diversity of outer membrane proteins (Hashemolhosseini et al., 1994). Consequently, phage cocktails are prepared to broaden host range because the recognition of bacterial receptors is not universal (Chan et al., 2013). Phage cocktails are an amalgam of phages, each possessing different host ranges. Collectively, 
they expand the host-range to confer a broader spectrum of activity against infectious bacteria (Fig. 2). Additionally, phage cocktails prevent the rapid emergence of phage-resistant strains. Bacteria may evolve resistance to a specific phage, but it would be unrealistic for the bacteria to simultaneously develop multiple resistance mechanisms to a collective group of phages (Goodridge, 20Io; Tanji et al., 2005).

\section{Recent advances of phage therapy}

Currently, there are no commercially available therapeutic phages. The bulk of phage therapy research is concentrated in Eastern Europe and Soviet Union. The US and Europe lost much of their interest in phage therapy after the discovery of antibiotics, but Soviet research continued (Kutatekadze \& Revaz, 20IO). However, due to multidrug resistance, interest in phage therapy is increasing in the US and Europe leading to several clinical trials as shown in Table I. A Phase II clinical trial for phage therapy was recently completed by Wright et al., who reported long-term positive results for ear infections, burn wounds, and leg ulcers caused by Pseudomonas aeruginosa infections (Merril et al., 2003; Harper \& Enright, 20II). This demonstrates the effectiveness of phage therapeutics in P. aeruginosa, a bacterial species that has evolved multidrug resistance.

While there are no current therapeutic applications of phages, the United States Food and Drug Administration (FDA) has approved spraying meat with phages to eliminate Listeria monocytogenes, a highly virulent food-borne pathogen that was the primary cause of listeriosis, in August 2006 (Ramaswamy, 2007). ListShield is a phage cocktail containing six listeria-specific phages designed to attack a very broad spectrum of pathogenic L. monocytogenes strains. The phage preparation is sprayed on food surfaces, and later consumed without any significant side effects. Strict phage-host specificity targets only pathogenic L. monocytogenes strains. At the very least, this should demonstrate that direct human use of phages is likely to be very safe (Mai et al., 20IO).

\section{Current therapeutic treatment options}

Excessive use of antibiotics has reinforced selective pressure for antibiotic resistance, accelerating the emergence of these "superbugs" (Drawz \& Bonomo, 20IO). As a result, our antibiotic armamentarium is exhausted, severely restricting our ability to respond to new multidrug-resistant threats. The antibiotic pipelines are nearly dry, failing to deliver novel antibiotics to match today's multidrug resistance crisis. Only two new classes of antibiotics have been developed in the past 3 decades (Theuretzbacher, 20I2; Gupta \& Nayak, 20I4). Treatment options for multidrug-resistant infections are extremely limited. While most isolates remain susceptible to colistin and tigecycline, their therapeutic viability is questionable. Recurring infection after treatment with tigecycline increased the MIC (minimum inhibitory concentration) significantly (Daly et al., 2007); colistin usage should be limited due to its neurotoxicity and nephrotoxicity (Zavascki et al., 2007).

\section{Targeting Klebsiella pneumoniae carbapenemase (KPC)-producing K. pneumoniae with a phage cocktail}

Klebsiella pneumoniae carbapenemase (KPC)-producing K. pneumoniae are becoming a growing menace due to their resistance to $\beta$-lactam antibiotics and nearly all commercially available antibiotics; this includes carbapenems, which are considered a drug of last resort against highly resistant microorganisms. The enzyme K. pneumoniae carbapenemase was first discovered in $K$. pneumoniae but has since been 
found in other enterobacteriaceae. More than two million people are affected by antibiotic-resistant infections with at least 23,000 deaths. They are a leading cause of nosocomial infections. The first KPCproducing $K$. pneumoniae outbreaks were reported in hospitals in New York City before spreading globally to foreign healthcare environments (Bratu et al., 2005; Leavitt et al., 2007). KPC-producing $K$. pneumoniae are responsible for an estimated 9,000 drug-resistant infections per year and 520 attributed deaths (Bell et al., 2009). The attributable mortality rate due to KPC-producing $K$. pneumoniae bacteremia infections is $50 \%$, the highest reported so far for any microorganism causing bacteremia, requiring more intensive patient care and exhausting the current antibiotic arsenal (Borer et al., 2009; Patel et al., 2008).

\section{Hypothesis}

A phage preparation consisting of multiple phages specific to $K$. pneumoniae will successfully infect and lyse bacterial host cells of various KPC-producing $K$. pneumoniae strains, due to the preparation's broad spectrum of activity.

\section{Primary Objectives}

- Screen fecal material obtained from the San Luis Obispo Wastewater Treatment facility as a source of $K$. pneumoniae-specific phage and inoculate with several representative strains of $K$. pneumoniae to perform a plaque assay.

- Isolate several phages based on their ability to produce lawns on the KPC-producing K. pneumoniae host bacterium and identify and classify the phages.

- Propagate phages until the desired yield of amplification is attained, and then purify and precipitate phages for use in in vitro assessments of its effectiveness.

- Test effectiveness by adding the phage cocktail to all KPC-producing K. pneumoniae strains grown in culture, and assessing the phage cocktail's ability to lyse the KPC-producing K. pneumoniae cultures.

\section{Methods}

Bacterial strain. Due to the moderate threat posed by the resistant $K$. pneumoniae strains, all microbial cultures will be handled according to the appropriate safety procedures and precautions corresponding to Biosafety Level 2 (Chosewood et al., 2009). Several representative strains (BAA-I705, BAA-I900, BAA-2078, BA-2342, BAA-2343 and BAA-2344) of $K$. pneumoniae that produce the enzyme KPC will be ordered from the American Type Culture Collection (ATCC) for phage isolation, amplification, and evaluation. All strains are resistant to a broad spectrum of antibiotics, including carbapenems, making them ideal models for the assessment of a phage cocktail. The strains will be grown at $37^{\circ} \mathrm{C}$ in the supplied ATCC ${ }^{\oplus}$ Medium. This media contains nutrient agar (beef extract, peptone, and agar). The strains will be stocked in nutrient broth containing $40 \%$ glycerol and stored at $-80^{\circ} \mathrm{C}$. Cultures will be stored at $4^{\circ} \mathrm{C}$ and transferred periodically. 
Bacteriophage isolation and enrichment. Fecal material will be screened as a source for K. pneumoniae strain-specific phages. Fecal samples will be obtained from the San Luis Obispo Wastewater Treatment facility over a four month period. Portions of the samples will be added to volumes of early log-phase $K$. pneumoniae (grown at $37^{\circ} \mathrm{C}$ ) containing Io $\mathrm{mM} \mathrm{CaCl}$. These cultures will be incubated overnight at $37^{\circ} \mathrm{C}$ with shaking (200 rpm). Samples will be withdrawn and centrifuged in a microcentrifuge for $5 \mathrm{~min}$. The supernatant will be removed and passed through a sterilized filter before being subjected to a plaque assay using $K$. pneumoniae as the host. Plaques appearing on lawns on the host bacteria will be further processed to achieve desired phage stock of phage isolate candidates (Bourdin et al., 2OI3).

Phage morphology characterization. Identification and classification of phage candidates will be determined by observing the morphology of the phages by transmission electron microscopy. Phage particles will be sedimented at 25,000 g for $60 \mathrm{~min}$. using a centrifuge, and then the phage particles will be washed twice in ammonium acetate $(\mathrm{pH} 7.2)$ and deposited on copper grids provided with carbon-coated Formvar films. They will be stained with $2 \%$ potassium phosphotungstate ( $\mathrm{pH} 7.2$ ) and examined in a Philips EM 300 transmission electron microscope (Bourdin et al., 2013).

Phage amplification and purification. Phage candidates will be amplified in a 2-L Erlenmeyer flask filled with $4 \mathrm{~L}$ of $\mathrm{LB}$ medium (grown at $37^{\circ} \mathrm{C}$ and $\mathrm{pH}$ maintained at 7.0 with $\mathrm{NaOH}$ IO\%). The medium will be inoculated with $3 \%$ of an overnight $K$. pneumoniae culture. At OD600 of 0.5 , the bacteria will be infected with the phage lysates at a multiplicity of infection of $\mathrm{I}$. The flask will be placed in an incubator and shaken for 7 hours (400 rpm). Phages from the combined phage lysates will be separated from organic debris by low speed centrifugation, and then by additional ultracentrifugation to pellet the phages. The pellet will be re-suspended in phage buffer ( $150 \mathrm{mM} \mathrm{NaCl}$ and ${ }_{10} \mathrm{mM} \mathrm{Mg} \mathrm{SO}_{4}$ in sterile water at $\mathrm{pH}$ 7.2) (Bourdin et al., 2013).

Phage cocktail testing. A $50 \mu \mathrm{l}$ of $K$. pneumoniae ATCC BAA-1705, BAA-1900, BAA-2078, BA-2342, BAA-2343 and BAA-2344 overnight culture will be inoculated into $5 \mathrm{ml}$ of LB broth and incubated with shaking at $37^{\circ} \mathrm{C}$. The change of culture turbidity of OD660 will be automatically recorded by photo recorder without fractionating the sample from the culture. When the culture reaches an OD660 of o.I, the phage cocktail will be added at a multiplicity of infection of Io. The subsequent OD660 of the culture will be monitored. All assays will be performed in triplicate to confirm reproducibility and assess the effectiveness of the phage cocktail (Tanji et al., 2005). The effectiveness of the phage cocktail will be determined by measuring the optical density of the culture before and after treatment with the phage cocktail.

Statistical analysis. The OD660 value of the bacterial culture before application of the phage cocktail will be recorded for each strain. After the addition of the phage cocktail, the subsequent OD660 value of the bacterial culture will be recorded. The mean OD66o values of the triplicate assay of all bacterial strains will be used for data analysis. An independent two-sample t-test will be performed to determine whether there is significant bacterial reduction after the application of a phage therapy. The data set is assumed to be equal in sample size and variance because it is the same population of bacteria being tested. Calculations will be performed in $\mathrm{R}$, a free software programming for statistical computing. A significant difference will suggest that application of a phage cocktail is effective in bacterial reduction, and the opposite is true if there is no significant difference. 


\section{Timeline}
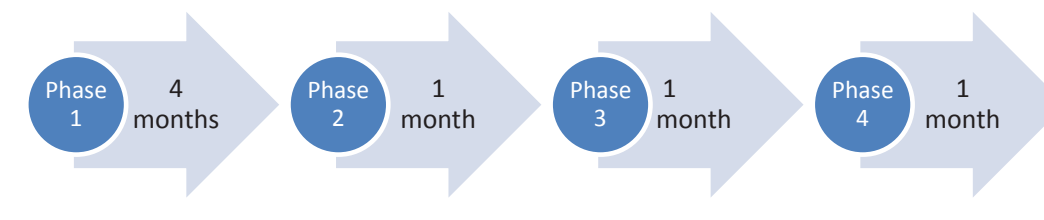

\begin{tabular}{|c|c|}
\hline Phase 1 & $\begin{array}{l}\text { A major portion of the research will be focused on screening fecal samples and selecting } \\
\text { phages from an environmental source for use in a phage cocktail. Once appropriate phage } \\
\text { candidates have been selected based on their ability to lyse KPC-producing K. pneumoniae } \\
\text { strains, they will be enriched, amplified and purified. }\end{array}$ \\
\hline Phase 2 & $\begin{array}{l}\text { Candidate phages will be identified and classified based on their morphology using } \\
\text { transmission electron microscopy. This is to ensure they are suitable for use and do not } \\
\text { produce any toxins. }\end{array}$ \\
\hline Phase 3 & $\begin{array}{l}\text { Candidate phages will be amplified and purified to produce the final phage cocktail } \\
\text { product. }\end{array}$ \\
\hline Phase 4 & $\begin{array}{l}\text { The effectiveness of the phage cocktail will be tested by administering the phage cocktail } \\
\text { to all cultures of KPC-producing K. pneumoniae strains, and assessing the optical density } \\
\text { of the broth culture as a proxy of bacterial lysis. }\end{array}$ \\
\hline
\end{tabular}

\section{Budget}

\begin{tabular}{|l|rr|}
\hline Salary and Wages & $\$ 2800$ \\
\hline Equipment & & $\$ 0$ \\
\hline Materials and Supplies & $\$ 1,770$ \\
\hline Travel Costs & $\$ 0$ \\
\hline Total Direct Costs & $\$ 4,570$ \\
& & \\
\hline
\end{tabular}

The undergraduate student researcher will be paid $\$$ Io per hour and will spend to hours a week for 7 months on this research project. The student will therefore be paid a total of $\$ 2800$. KPC-producing K. pneumoniae strains ordered from ATCC will cost $\$ 295$ each, thus six strains of the bacteria will cost $\$$ \$,770 in total. All other equipment such as a spectrophotometer, ultracentrifuge, and a transmission electron microscope will be available for use due to collaboration with the Biology and Biomedical Engineering Department at California Polytechnic State University, San Luis Obispo. There will be no travel costs associated with this research. 


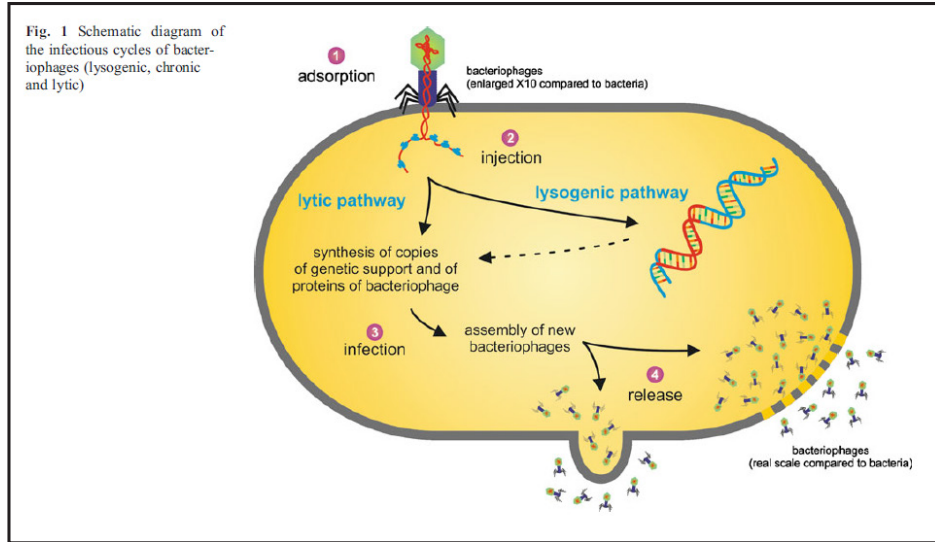

Figure I. This shows the infectious life cycle of bacteriophages. It begins with adsorption, injection of genetic material, infection, and then the release of newly assembled bacteriophages.

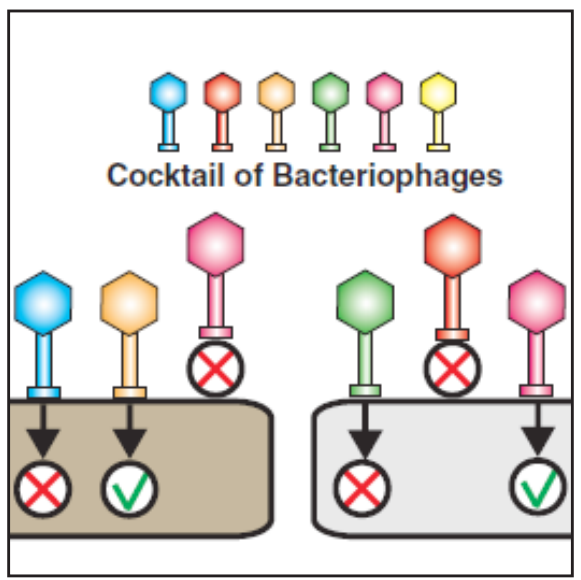

Figure 2. This illustrates the benefit of using a cocktail of bacteriophages by broadening the host-range to confer greater infectivity against multiple bacterial strains.

\begin{tabular}{|c|c|c|c|c|c|c|c|}
\hline Target & Entity & Characteristics & Dosing & Phages ${ }^{+}$ & Reduction ${ }^{t}$ & Comments & Ref. \\
\hline E. coli & Cattle & Colonization & Oral & $\mathrm{m} \times 4$ & - & $\begin{array}{l}\text { Marginally positive } \\
\text { reductions }\end{array}$ & [97] \\
\hline P. aeruginosa & Human & Otitis & Topical & $u \times 6$ & $10^{\prime}$ & $\begin{array}{l}\text { Phase VII clinical trial; } \\
\text { indication of efficacy } \\
\text { with no adverse effects }\end{array}$ & [46] \\
\hline E. coli & Food & - & - & $\mathrm{m} \times 3$ & - & Reductions up to $100 \%$ & [79] \\
\hline $\begin{array}{l}\text { Salmonella } \\
\text { enteritidis ser. } \\
\text { Enteritidis }\end{array}$ & Poultry & Colonization & Spray & $u \times 3$ & $10^{17}$ & $\begin{array}{l}\text { Phage treatment prior } \\
\text { to bacterial inoculation }\end{array}$ & [98] \\
\hline $\begin{array}{l}\text { 5. enteritidis ser. } \\
\text { Enteritidis }\end{array}$ & Poultry & Colonization & Oral & $u \times 4$ or $u \times 45$ & - & $\begin{array}{l}\text { Substantial early } \\
\text { reductions in } \\
\text { colonization }(45-100 \%) \\
\text { but less later }(15 \%)\end{array}$ & [99] \\
\hline P. aeruginosa & Murine & Burn wound & im., ip. or sc. & $u \times 3$ & - & $\begin{array}{l}12 \% \text { mortality with } \\
\text { phage treatment (ip.) } \\
\text { and } 94 \% \text { without }\end{array}$ & [43] \\
\hline \multicolumn{8}{|c|}{ 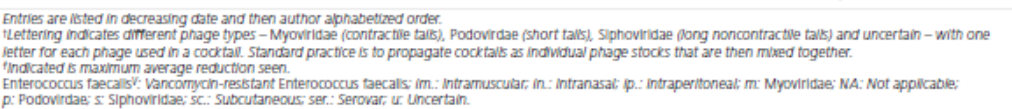 } \\
\hline
\end{tabular}

Table I. This table highlights several recent investigations of bacterial reduction using phage cocktails. 


\section{References}

Abedon, Stephen T., and Cameron Thomas-Abedon. (2010). "Phage therapy pharmacology." Current Pharmaceutical Biotechnology II(I), 28-47.

Ambler, R. P., et al. (199I). “A standard numbering scheme for the class A beta-lactamases." Biochemical Journal 276, 269.

Bell, B., et al. (2009). "Antibiotic Resistance Threats in the United States, 2013." Center for Disease Control and Prevention.

Borer, Abraham, et al. (2009). "Attributable Mortality Rate for Carbapenem-Resistant Klebsiella pneumoniae Bacteremia." Infection Control and Hospital Epidemiology 30(го), 972-976.

Bourdin, Giles, et al. (2013). "Amplification and purification of T4-like Escherichia coli Phages for Phage Therapy: from Laboratory to Pilot Scale." Appl. Environ. Microbiol. 80(4), I469-I476.

Bratu, S., et al. (2005). "Rapid spread of carbapenem-resistant Klebsiella pneumoniae in New York City - A new threat to our antibiotic armamentarium." Archives of Internal Medicine I65(I2), I430-I435.

Carlton, Richard M. (1999). "Phage therapy: past history and future prospects." Archivum Immunologiae Et Therapiae Experimentalis-English Edition 47, 267-274.

Chan, Benjamin K., Stephen T. Abedon, and Catherine Loc-Carrillo. (2013). "Phage cocktails and the future of phage therapy." Future Microbiology 8(6), 769-783.

Chosewood, Casey L., et al. (2009). Biosafety in Microbiological and Biomedical Laboratories. [PDF document]. Retrieved from Center for Disease Control and Prevention: http://www.cdc.gov/biosafety/publications/bmbl5/.

Daly, Michael W., et al. (2007). "Tigecycline for Treatment of Pneumonia and Empyema Caused by Carbapenemase-Producing Klebsiella pneumoniae." Pharmacotherapy: The Journal of Human Pharmacology and Drug Therapy 27(7), I052-I057.

Drawz, Sarah M, and Robert Bonomo. (20I0). “Three Decades of beta-Lactamase Inhibitors.” Clinical Microbiology Reviews 23(I), I60.

Goodridge, Lawrence D. (2010). “Designing phage therapeutics." Current Pharmaceutical Biotechnology II(I), I5-27.

Gupta, Ragini, and Yogendra Prasad. (20II). "Efficacy of polyvalent bacteriophage P-27/HP to control multidrug resistant Staphylococcus aureus associated with human infections." Current Microbiology 62(I), 255-260.

Gupta, S. K., and R. P. Nayak. (20I4). "Dry antibiotic pipeline: Regulatory bottlenecks and regulatory reforms." Journal of Pharmacology and Pharmacotherapeutics $5(\mathrm{I}), 4$.

Hanlon, Geoffrey William. (2007). "Bacteriophages: an appraisal of their role in the treatment of bacterial infections." International Journal of Antimicrobial Agents 30(2), II8-I28.

Harper, D. R., and M. C. Enright. (20II). "Bacteriophages for the treatment of Pseudomonas aeruginosa infections." Journal of Applied Microbiology $\operatorname{III}(\mathrm{I}), \mathrm{I}-7$.

Hashemolhosseini, S., Montag, D., Kramer, L., Henning, U. (1994). "Determinants of receptor specificity of coliphages of the T4 family: A chaperone alters the host range." J. Mol. Biol 24I(4), 524-533.

Henn, Matthew R., et al. (2010). "Analysis of high-throughput sequencing and annotation strategies for phage genomes." PLoS One 5(2), e9o83.

Hidron, Alicia I., et al. (2008). "Antimicrobial-resistant pathogens associated with healthcare-associated infections: annual summary of data reported to the National Healthcare Safety Network at the Centers for Disease Control and Prevention, 2006-2007." Infection Control and Hospital Epidemiology 29(II), 996-IOII.

Hyman, Paul, and Stephen T. Abedon. (2010). "Bacteriophage host range and bacterial resistance." Advances in Applied Microbiology 70, $217-248$.

Kitchel, Brandon, et al. (2009). "Molecular epidemiology of KPC-producing Klebsiella pneumoniae isolates in the United States: clonal expansion of multilocus sequence type 258." Antimicrobial Agents and Chemotherapy 53(8), 3365-3370.

Kramberger, Petra, et al. (2010). "Purification of the Staphylococcus aureus bacteriophages VDX-io on methacrylate monoliths." Journal of Virological Methods I66(I), 60-64.

Kutateladze, Mzia, and Revaz Adamia. (2010). "Bacteriophages as potential new therapeutics to replace or supplement antibiotics." Trends in Biotechnology 28(I2), 591-595. 
Kutter, Elizabeth, et al. (20I0). "Phage therapy in clinical practice: treatment of human infections." Current Pharmaceutical Biotechnology iI(I), 69-86.

Queenan, Anne Marie, and Karen Bush. (2007). “Carbapenemases: the versatile $\beta$-lactamases.” Clinical Microbiology Reviews 20(3), $440-458$.

Leavitt, Azita, et al. (2007). "Emergence of KPC-2 and KPC-3 in carbapenem-resistant Klebsiella pneumoniae strains in an Israeli hospital." Antimicrobial agents and chemotherapy 5I(8), 3026-3029.

Lledo, W., et al. (2009). "Guidance for control of infections with carbapenem-resistant or carbapenemase-producing Enterobacteriaceae in acute care facilities." Morbidity and Mortality Weekly Report 58(Io), 256-258

Loc-Carrillo, Catherine, and Stephen T. Abedon. (2OII). "Pros and cons of phage therapy." Bacteriophage I(2), III-II4.

Merril, Carl R., Dean Scholl, and Sankar L. Adhya. (2003). “The prospect for bacteriophage therapy in Western medicine.” Nature Reviews Drug Discovery 2(6), 489-497.

Nordmann, Patrice, Gaelle Cuzon, and Thierry Naas. (2009). "The real threat of Klebsiella pneumoniae carbapenemase-producing bacteria." The Lancet Infectious Diseases 9(4), 228-236.

Oliveira, A., et al. (2009). "Isolation and characterization of bacteriophages for avian pathogenic E. coli strains." Journal of Applied Microbiology I06(6), I919-1927.

Patel, Gopi, et al. (2008). "Outcomes of carbapenem-resistant Klebsiella pneumoniae infection and the impact of antimicrobial and adjunctive therapies." Infection Control and Hospital Epidemiology 29(I2), I099-IIo6.

Paterson, David L., and Robert A. Bonomo. (2005). "Extended-spectrum $\beta$-lactamases: a clinical update.” Clinical Microbiology Reviews I8(4), 657-686.

Phillips M, Sharma S. (2009). Clinical outcomes of infections caused by KPC-producing organisms; NIH Workshop on ESKAPE Pathogens; Bethseda, MD.

Ramaswamy, Vidhya, et al. (2007). "Listeria-review of epidemiology and pathogenesis." Journal of Microbiology Immunology and Infection $40 . \mathrm{I}$, 4.

Skurnik, Mikael, Maria Pajunen, and Saija Kiljunen. "Biotechnological challenges of phage therapy." Biotechnology Letters 29(7), 995-I003.

Tanji, Yasunori, et al. (2005). "Therapeutic use of phage cocktail for controlling Escherichia coli Ois7: $\mathrm{H}_{7}$ in gastrointestinal tract of mice." Journal of Bioscience and Bioengineering $100(3), 280-287$.

Theuretzbacher, Ursula. (2OI2). "Accelerating resistance, inadequate antibacterial drug pipelines and international responses." International Journal of Antimicrobial Agents 39(4), 295-299.

Volker, Mai et al. (20I0). "Bacteriophage Administration Reduces the Concentration of Listeria monocytogenes in the Gastrointestinal Tract and Its Translocation to Spleen and Liver in Experimentally Infected Mice." International Journal of Microbiology

Young, R. Y. (1992). “Bacteriophage lysis: mechanism and regulation.” Microbiological Reviews 56(3), 430.

Zavascki, Alexandre Prehn, et al. (2007). "Polymyxin B for the treatment of multidrug-resistant pathogens: a critical review." Journal of Antimicrobial Chemotherapy 60(6), I206-I2I5. 


\section{ABOUT THE AUTHOR}

STEVEN LIU is a senior biological sciences student and microbiology minor. After graduation, he wishes to attend graduate school and then apply to an epidemiology or public health $\mathrm{PhD}$ program. His ideal career would be to join the Epidemic Intelligence Service with the Center for Disease Control and Prevention (CDC). Steven finds it amazing that the life sciences hold so much potential to benefit the health of humans. "Even after so many groundbreaking medical breakthroughs and advances, there still remains so much potential in the life sciences to improve the overall health of communities worldwide," Steven said. He finds the piece he submitted to Symposium on antibiotic resistance is a serious topic that has yet to be fully understood. Steven believes that the impact of antibiotic resistance is important because many current medical processes and procedures require antibiotics. "I hope my piece will shed more light on antibiotic resistance and the severity of the situation," he said. The labs and professors, such as Dr. Winstead and Dr. Black, in the College of Science and Math have inspired him to delve into the microbial world and the explore the huge impact that such microorganisms may have on our lifestyle. 